\title{
The first report of Daldinia eschscholtzii as an endophyte from leaves of Musa sp. (Musaceae) in Thailand
}

\section{Samarakoon SMBC ${ }^{1,2}$, Samarakoon $\mathrm{MC}^{2,5}$, Aluthmuhandiram $\mathrm{JVS}^{1,2,6}$, Wanasinghe $\mathrm{DN}^{3,4}$ and Chomnunti $\mathbf{P}^{1,2^{*}}$}

${ }^{1}$ School of Science, Mae Fah Luang University, Chiang Rai 57100, Thailand

${ }^{2}$ Center of Excellence in Fungal Research, Mae Fah Luang University, Chiang Rai 57100, Thailand

${ }^{3}$ Key Laboratory for Plant Biodiversity and Biogeography of East Asia (KLPB), Kunming Institute of Botany, Chinese Academy of Science, Kunming 650201, Yunnan, China

${ }^{4}$ World Agro Forestry Centre, East and Central Asia, 132 Lanhei Road, Kunming 650201, Yunnan, China

${ }^{5}$ Department of Biology, Faculty of Science, Chiang Mai University, Chiang Mai 50200, Thailand

${ }^{6}$ Beijing Key Laboratory of Environment Friendly Management on Fruit Diseases and Pests in North China, Institute of Plant and Environment Protection, Beijing Academy of Agriculture and Forestry Sciences, Beijing 100097, China

Samarakoon SMBC, Samarakoon MC, Aluthmuhandiram JVS, Wanasinghe DN, Chomnunti P 2019 - The first report of Daldinia eschscholtzii as an endophyte from leaves of Musa sp. (Musaceae) in Thailand. Asian Journal of Mycology 2(1), 183-197, Doi 10.5943/ajom/2/1/9

\begin{abstract}
There has been increasing research interest in the isolation of fungal endophytes from different hosts or tissue types of the same host from many tropical regions. However, there have been few studies conducted on musaceous endophytes in Thailand. In this study, we provide the first report of Daldinia eschscholtzii (Hypoxylaceae) as an endophyte isolated from symptomless fresh leaves of Musa sp. (Musaceae) in northern Thailand. In addition, this is the first record of Daldinia from Musaceae and the second report of D. eschscholtzii from monocotyledons. Fungi isolates are illustrated, described and subjected to LSU-ITS-RPB2-BTUB concatenated phylogenies using maximum likelihood and Bayesian analysis with an updated tree of Hypoxylaceae.
\end{abstract}

Keywords - Banana - Host - Hypoxylaceae - Monocots - Record

\section{Introduction}

Fungi exhibit various nutritional modes such as endophytes, saprobes and pathogens in different hosts and habitats (Wang et al. 2005, Hyde et al. 2018, Tibpromma et al. 2018, Jayawardena et al. 2019). Endophytes inhabit living and healthy tissues without causing apparent disease symptoms (Schulz \& Boyle 2006). Musa sp. (banana) is a tropical monocotyledonous plant in family Musaceae. Several endophytic fungi have been recorded from Musa species in Australia (Brown et al. 1998), Brazil (Pereira et al. 1999), Central America (Pocasangre et al. 1999), China (Cao et al. 2002, 2003) and Hong Kong (Brown et al. 1998). Acremonium Link (Pocasangre et al. 1999, Cao et al. 2003), Bipolaris Shoemaker (Zakaria \& Aziz 2018), Colletotrichum Corda (Brown et al. 1998, Cao et al. 2003), Fusarium Link, Nigrospora Zimm. (Brown et al. 1998), Pestalotiopsis Steyaert, Phoma Fr., Trichoderma Pers. and Verticillium Nees (Pocasangre et al. 1999, Cao et al. 2003) were the common genera that found as fungal endophytes from Musa sp. Cao et al. (2002) 
recorded Alternaria Nees, Aspergillus P. Micheli, Aureobasidium Viala \& G. Boyer, Cephalosporium Corda, Cladosporium Link, Deightoniella S. Hughes, Gloeosporium Desm. \& Mont., Myxosporium Link, Sarcinella Sacc., Sphaceloma de Bary and Uncinula Lév. from Musa sp. In addition, there are numerous xylariaceous taxa from Xylariaceae were also recorded as endophytes from Musa sp. (Brown et al. 1998, Pereira et al. 1999).

Photita et al. (2001, 2004) documented fungal endophytes of wild M. acuminata from northern Thailand including Guignardia Viala \& Ravaz and Periconiella Sacc. The pathogenicity of endophytic Cladosporium musae E.W. Mason, Colletotrichum gloeosporioides (Penz.) Penz. \& Sacc., Deightoniella torulosa (Syd.) M.B. Ellis, Guignardia cocoicola Punith., Neocordana musae (Zimm.) Hern.-Restr. \& Crous and Periconiella musae Stahel ex M.B. Ellis was evaluated and D. torulosa was identified to cause disease symptoms in Musa leaves in vitro (Photita et al. 2004).

The genus Daldinia (Hypoxylaceae) was introduced by Cesati \& De Notaris (1863). Currently the genus accommodates around 50 taxa and distributed in terrestrial and marine habitats. The genus has frequently recorded from tropics compared to temperate regions with saprobic and endophytic nutritional modes (Johannesson et al. 2001, Guidot et al. 2003, Nugent 2004, Stadler et al. 2014, Wijayawardene et al. 2017).

Traditionally Daldinia was accommodated in Xylariaceae. Stadler et al. (2014) provided a detailed comprehensive study on the genus using multiple taxonomic approaches including type material studies, sexual/asexual morphs and the cultural characteristics coupled with Scanning Electron Microscopic (SEM) observations. HPLC chemical profiles, UV visible spectroscopy, mass spectroscopy and 5.8S/ITS nrDNA phylogenetic studies also were used.

Wendt et al. (2018) constructed a multigene phylogeny for stromatic Xylariales species based on ITS, LSU, RPB2 and BTUB sequence data. As a result, Xylariaceae was segregated into many major clades and Daldinia was accommodated in Hypoxylaceae. The morphological characteristics such as stromatal pigments and nodulisporium-like asexual morphs further supported the placement of Daldinia in Hypoxylaceae by Wendt et al. (2018). After Stadler et al. (2014) the genus was updated with two more new species with D. korfii Sir \& C. Lambert (Sir et al. 2016) and D. subvernicosa Srikit. et al. (Wongkanoun et al. 2019).

Daldinia eschscholtzii was established in 1820 by Ehrenberg as Sphaeria eschscholtzii. Apart from the terrestrial plants, D. eschscholtzii has also been recorded in marine algae, mangroves, nails, skin and human blood (Karnchanatat et al. 2007, Zhang et al. 2008, Tarman et al. 2012, Kongyen et al. 2015, Ng et al. 2016, Helaly et al. 2018). The sexual morph of D. eschscholtzii appears as a turbinate to placentiform shaped stromata in warm and exposed habitats frequently on dead or decaying woody substrates of several angiosperms and few gymnosperms (Stadler et al. 2014). The asexual morph of $D$. eschscholtzii resembles hyaline hyphomycetes and the conidiogenous structures of $D$. eschscholtzii represent a nodulisporium-like branching pattern (Stadler et al. 2014, Wijayawardene et al. 2017).

In this study, we collected fresh symptomless leaf samples of Musa sp. from Chiang Rai Phu Chi Fah and Phayao in northern Thailand and isolated three endophytic D. eschscholtzii strains. We present the first report of $D$. eschscholtzii as a foliicolous endophyte in Musa sp. In addition, this is the first endophytic report of genus Daldinia in Musa sp. and the first molecular and morphological justification of the occurrence of $D$. eschscholtzii in monocotyledons.

\section{Materials and methods}

\section{Fungal isolation morphological characterization}

Symptomless fresh Musa leaves were collected from Chiang Rai, Phu Chi Fah and Phayao in northern Thailand. Small pieces $\left(<5 \mathrm{~mm}^{2}\right)$ from the leaf tissues were separated using a sterilized scalpel, disinfected in 75\% ethanol for $1 \mathrm{~min}$, rinsed three times in sterile distilled water, dried and placed on potato dextrose agar ( $2 \%$ potato dextrose agar PDA). The plates were incubated at $25^{\circ} \mathrm{C}$ for at least 5 days until fungi developed. Hyphal tips were transferred to fresh PDA plates using a sterilized scalpel and incubated at $25^{\circ} \mathrm{C}$ temperature and light conditions. Asexual morphs were 
observed from the cultures after 14 days using a Motic SMZ 168 Stereo Zoom Microscope. Observed conidia were used for single spore isolations and incubated at $25^{\circ} \mathrm{C}$. Growth rates and cultural characteristics were documented. Living and dry cultures were deposited in the Mae Fah Luang University Culture Collection (MFLUCC) and Mae Fah Luang University Herbarium (MFLU), Chiang Rai, Thailand respectively.

\section{DNA extraction and PCR amplification}

Fungal isolates were grown on potato dextrose agar (PDA) for 4 weeks at $25^{\circ} \mathrm{C}$ and total genomic DNA was extracted from 50 to $100 \mathrm{mg}$ of axenic mycelium of the growing culture (Wanasinghe et al. 2018). Mycelium was ground to a fine powder with liquid nitrogen and fungal DNA was extracted using the Biospin Fungus Genomic DNA Extraction Kit-BSC14S1 (BioFlux, P.R. China) according to the instructions of the manufacturer. Four gene regions as ITS, partial 28S large subunit (LSU), partial beta-tubulin (BTUB) and partial second largest subunit of the DNAdirected RNA polymerase II (RPB2) were amplified using ITS5/ITS4 (White et al. 1990), LR0R/LR5 (Vilgalys \& Hester 1990), T1/T2 (O’Donnell \& Cigelnik 1997) and fRPB2-5f/fRPB27cR (Liu et al. 1999) primers respectively.

A total volume of $26.5 \mu \mathrm{l}$ PCR mixture contained TaKaRa E-Taq DNA polymerase $0.3 \mu 1$, $12.5 \mu \mathrm{l}$ of $2 \times$ PCR buffer with $2.5 \mu \mathrm{l}$ of dNTPs, $1 \mu \mathrm{l}$ of each primer, $9.2 \mu \mathrm{l}$ of double-distilled water and 100-500 ng of DNA template followed by thermal cycle programs described by Wanasinghe et al. (2014) and Wendt et al. (2018). All the PCR products were visualized by staining with ethidium bromide (EtBr) on $1.2 \%$ agarose gels. Successful PCR products were purified according to the manufacturer's instructions of a Qiagen purification kit (Qiagen, USA) and sequenced in Sunbiotech Company, Beijing, China.

\section{Sequencing and sequence alignment}

Obtained sequences were subjected to BLAST search in GenBank (https://blast.ncbi.nlm.nih.gov/Blast.cgi). BLAST search results and initial morphological studies have supported that our isolates have belonged to Hypoxylaceae. Other sequences used in the analyses were obtained from GenBank based on recently published data (Stadler et al. 2014, Wendt et al. 2018) (Table 1). The single gene alignments were automatically done by MAFFT v. 7.036 (http://mafft.cbrc.jp/alignment/server/index.html, Katoh et al. 2017) using the default settings and later refined where necessary, using BioEdit v. 7.0.5.2 (Hall 1999).

Table 1 Taxa that used in the phylogenetic analysis of $D$. eschscholtzii with the corresponding GenBank Accession numbers. Type strains are superscripted with $\mathrm{T}$ and newly generated strains are indicated bold.

\begin{tabular}{|c|c|c|c|c|c|c|}
\hline \multirow[t]{2}{*}{ Species } & \multirow{2}{*}{$\begin{array}{l}\text { Strain } \\
\text { number }\end{array}$} & \multicolumn{4}{|c|}{ GenBank accession numbers } & \multirow[t]{2}{*}{ References } \\
\hline & & ITS & LSU & RPB2 & BTUB & \\
\hline $\begin{array}{l}\text { Annulohypoxylon } \\
\text { atroroseum }\end{array}$ & ATCC 76081 & AJ390397 & KY610422 & KY624233 & DQ840083 & $\begin{array}{l}\text { Kuhnert et } \\
\text { al. (2014), } \\
\text { Wendt et al. } \\
\text { (2018) }\end{array}$ \\
\hline A. michelianum & CBS 119993 & KX376320 & KY610423 & KY624234 & KX271239 & $\begin{array}{l}\text { Kuhnert et } \\
\text { al. (2014), } \\
\text { Wendt et al. } \\
\text { (2018) }\end{array}$ \\
\hline A. moriforme & CBS 123579 & KX376321 & KY610425 & KY624289 & KX271261 & $\begin{array}{l}\text { Kuhnert et } \\
\text { al. (2014), } \\
\text { Wendt et al. } \\
\text { (2018) }\end{array}$ \\
\hline A. nitens & $\begin{array}{l}\text { MFLUCC 12- } \\
0823\end{array}$ & KJ934991 & KJ934992 & KJ934994 & KJ934993 & $\begin{array}{l}\text { Daranagama } \\
\text { et al. (2015) }\end{array}$ \\
\hline
\end{tabular}


Table 1 Continued.

\begin{tabular}{|c|c|c|c|c|c|c|}
\hline \multirow[t]{2}{*}{ Species } & \multirow{2}{*}{$\begin{array}{l}\text { Strain } \\
\text { number }\end{array}$} & \multicolumn{4}{|c|}{ GenBank accession numbers } & \multirow[t]{2}{*}{ References } \\
\hline & & ITS & LSU & RPB2 & BTUB & \\
\hline A. stygium & MUCL 54601 & KY610409 & KY610475 & KY624292 & KX271263 & $\begin{array}{l}\text { Wendt et al. } \\
\text { (2018) }\end{array}$ \\
\hline Daldinia andina $^{\mathbf{T}}$ & CBS 114736 & - & KY610430 & KY624239 & КС977259 & $\begin{array}{l}\text { Kuhnert et } \\
\text { al. (2014), } \\
\text { Wendt et al. } \\
\text { (2018) }\end{array}$ \\
\hline D. bambusicola ${ }^{\mathbf{T}}$ & CBS 122872 & KY610385 & KY610431 & KY624241 & AY951688 & $\begin{array}{l}\text { Hsieh et al. } \\
\text { (2005), } \\
\text { Wendt et al. } \\
\text { (2018) }\end{array}$ \\
\hline D. caldariorum & MUCL 49211 & AM749934 & KY610433 & KY624242 & КС977282 & $\begin{array}{l}\text { Bitzer et al. } \\
\text { (2008), } \\
\text { Kuhnert et } \\
\text { al. (2014), } \\
\text { Wendt et al. } \\
\text { (2018) }\end{array}$ \\
\hline D. concentrica & CBS 113277 & AY616683 & KY610434 & KY624243 & КС977274 & $\begin{array}{l}\text { Triebel et al. } \\
\text { (2005), } \\
\text { Kuhnert et } \\
\text { al. (2014), } \\
\text { Wendt et al. } \\
\text { (2018) }\end{array}$ \\
\hline D. dennisii ${ }^{\mathbf{T}}$ & CBS 114741 & JX658477 & KY610435 & KY624244 & КС977262 & $\begin{array}{l}\text { Stadler et al. } \\
\text { (2014), } \\
\text { Kuhnert et } \\
\text { al. (2014), } \\
\text { Wendt et al. } \\
\text { (2018) }\end{array}$ \\
\hline D. eschscholtzii & MUCL 45435 & JX658484 & KY610437 & KY624246 & КС977266 & $\begin{array}{l}\text { Stadler et al. } \\
\text { (2014) }\end{array}$ \\
\hline D. eschscholtzii & CBS 113042 & JX658497 & - & - & - & $\begin{array}{l}\text { Stadler et al. } \\
\text { (2014) }\end{array}$ \\
\hline D. eschscholtzii & CBS 113047 & AY616684 & - & - & - & $\begin{array}{l}\text { Stadler et al. } \\
\text { (2014) }\end{array}$ \\
\hline D. eschscholtzii & CBS 116032 & JX658500 & - & - & - & $\begin{array}{l}\text { Stadler et al. } \\
\text { (2014) }\end{array}$ \\
\hline D. eschscholtzii & CBS 116035 & JX658498 & - & - & - & $\begin{array}{l}\text { Stadler et al. } \\
\text { (2014) }\end{array}$ \\
\hline D. eschscholtzii & CBS 116037 & JX658492 & - & - & - & $\begin{array}{l}\text { Stadler et al. } \\
\text { (2014) }\end{array}$ \\
\hline D. eschscholtzii & $\begin{array}{l}\text { CBS } \\
116037(2)\end{array}$ & JX658499 & - & - & - & $\begin{array}{l}\text { Stadler et al. } \\
\text { (2014) }\end{array}$ \\
\hline D. eschscholtzii & CBS 117735 & JX658480 & - & - & - & $\begin{array}{l}\text { Stadler et al. } \\
\text { (2014) }\end{array}$ \\
\hline D. eschscholtzii & CBS 117740 & JX658481 & - & - & - & $\begin{array}{l}\text { Stadler et al. } \\
\text { (2014) }\end{array}$ \\
\hline D. eschscholtzii & CBS 117741 & JX658491 & - & - & - & $\begin{array}{l}\text { Stadler et al. } \\
\text { (2014) }\end{array}$ \\
\hline D. eschscholtzii & CBS 122876 & JX658438 & - & - & - & $\begin{array}{l}\text { Stadler et al. } \\
\text { (2014) }\end{array}$ \\
\hline
\end{tabular}


Table 1 Continued.

\begin{tabular}{|c|c|c|c|c|c|c|}
\hline \multirow[t]{2}{*}{ Species } & \multirow{2}{*}{$\begin{array}{l}\text { Strain } \\
\text { number }\end{array}$} & \multicolumn{4}{|c|}{ GenBank accession numbers } & \multirow[t]{2}{*}{ References } \\
\hline & & ITS & LSU & RPB2 & BTUB & \\
\hline D. eschscholtzii & CBS 122877 & JX658439 & - & - & - & $\begin{array}{l}\text { Stadler et al. } \\
\text { (2014) }\end{array}$ \\
\hline D. eschscholtzii & CBS 122878 & JX658440 & - & - & - & $\begin{array}{l}\text { Stadler et al. } \\
\text { (2014) }\end{array}$ \\
\hline D. eschscholtzii & KC 1616 & JX658496 & - & - & - & $\begin{array}{l}\text { Stadler et al. } \\
\text { (2014) }\end{array}$ \\
\hline D. eschscholtzii & KC1699 & JX658490 & - & - & - & $\begin{array}{l}\text { Stadler et al. } \\
\text { (2014) }\end{array}$ \\
\hline D. eschscholtzii & MUCL 38740 & JX658493 & - & - & - & $\begin{array}{l}\text { Stadler et al. } \\
\text { (2014) }\end{array}$ \\
\hline D. eschscholtzii & MUCL 41777 & JX658486 & - & - & - & $\begin{array}{l}\text { Stadler et al. } \\
\text { (2014) }\end{array}$ \\
\hline D. eschscholtzii & MUCL 41778 & JX658494 & - & - & - & $\begin{array}{l}\text { Stadler et al. } \\
\text { (2014) }\end{array}$ \\
\hline D. eschscholtzii & MUCL 43508 & JX658495 & - & - & - & $\begin{array}{l}\text { Stadler et al. } \\
\text { (2014) }\end{array}$ \\
\hline D. eschscholtzii & MUCL 45434 & JX658484 & - & - & - & $\begin{array}{l}\text { Stadler et al. } \\
\text { (2014) }\end{array}$ \\
\hline D. eschscholtzii & MUCL 47965 & JX658482 & - & - & - & $\begin{array}{l}\text { Stadler et al. } \\
\text { (2014) }\end{array}$ \\
\hline D. eschscholtzii & $\begin{array}{l}\text { MFLUCC18- } \\
0177\end{array}$ & MK587659 & MK587746 & MK625010 & MK636689 & This study \\
\hline D. eschscholtzii & $\begin{array}{l}\text { MFLUCC19- } \\
0154\end{array}$ & MK587660 & MK587747 & MK625011 & MK636690 & This study \\
\hline D. eschscholtzii & $\begin{array}{l}\text { MFLUCC19- } \\
0153\end{array}$ & MK587661 & MK587748 & MK625012 & MK636691 & This study \\
\hline D. korfii ${ }^{\mathbf{T}}$ & STMA14089 & KY204020 & - & - & - & $\begin{array}{l}\text { Sir et al. } \\
\text { (2016) }\end{array}$ \\
\hline D. loculatoides ${ }^{\mathbf{T}}$ & CBS 113279 & MH862918 & KY610438 & KY624247 & KX271246 & $\begin{array}{l}\text { Johannesson } \\
\text { et al. (2000), } \\
\text { Stadler et al. } \\
\text { (2014) }\end{array}$ \\
\hline D. macaronesica ${ }^{\mathbf{T}}$ & CBS 113040 & KY610398 & KY610477 & KY624294 & KX271266 & $\begin{array}{l}\text { Wendt et al. } \\
\text { (2018) }\end{array}$ \\
\hline D. petriniae ${ }^{\mathbf{T}}$ & MUCL 49214 & - & KY610439 & KY624248 & КС977261 & $\begin{array}{l}\text { Bitzer et al. } \\
\text { (2008), } \\
\text { Kuhnert et } \\
\text { al. (2014), } \\
\text { Wendt et al. } \\
\text { (2018) }\end{array}$ \\
\hline D. placentiformis & MUCL 47603 & AM749921 & KY610440 & KY624249 & КС977278 & $\begin{array}{l}\text { Bitzer et al. } \\
\text { (2008), } \\
\text { Kuhnert et } \\
\text { al. (2014), } \\
\text { Wendt et al. } \\
\text { (2018) }\end{array}$ \\
\hline D. pyrenaica ${ }^{\mathbf{T}}$ & MUCL 53969 & KY610413 & - & KY624274 & KY624312 & $\begin{array}{l}\text { Wendt et al. } \\
\text { (2018) }\end{array}$ \\
\hline D. steglichii ${ }^{\mathrm{T}}$ & MUCL 43512 & KY610399 & KY610479 & KY624250 & KX271269 & $\begin{array}{l}\text { Wendt et al. } \\
\text { (2018) }\end{array}$ \\
\hline
\end{tabular}


Table 1 Continued.

\begin{tabular}{|c|c|c|c|c|c|c|}
\hline \multirow[t]{2}{*}{ Species } & \multirow{2}{*}{$\begin{array}{l}\text { Strain } \\
\text { number }\end{array}$} & \multicolumn{4}{|c|}{ GenBank accession numbers } & \multirow[t]{2}{*}{ References } \\
\hline & & ITS & LSU & RPB2 & BTUB & \\
\hline D. theissenii ${ }^{\mathrm{T}}$ & CBS 113044 & KY610388 & KY610441 & KY624251 & KX271247 & $\begin{array}{l}\text { Wendt et al. } \\
\text { (2018) }\end{array}$ \\
\hline D. vernicosa ${ }^{\mathbf{T}}$ & CBS 119316 & KY610395 & KY610442 & KY624252 & KC977260 & $\begin{array}{l}\text { Kuhnert et } \\
\text { al. (2014), } \\
\text { Wendt et al. } \\
\text { (2018) }\end{array}$ \\
\hline $\begin{array}{l}\text { Entonaema } \\
\text { liquescens }\end{array}$ & ATCC 46302 & KY610389 & KY610443 & KY624253 & KX271248 & $\begin{array}{l}\text { Wendt et al. } \\
\text { (2018) }\end{array}$ \\
\hline $\begin{array}{l}\text { Hypoxylon } \\
\text { carneum }\end{array}$ & MUCL 54177 & KY610400 & KY610480 & KY624297 & KX271270 & $\begin{array}{l}\text { Kuhnert et } \\
\text { al. (2014), } \\
\text { Wendt et al. } \\
\text { (2018) }\end{array}$ \\
\hline H. cercidicola & CBS 119009 & КС968908 & KY610444 & KY624254 & КС977263 & $\begin{array}{l}\text { Kuhnert et } \\
\text { al. (2014), } \\
\text { Wendt et al. } \\
\text { (2018) }\end{array}$ \\
\hline H. crocopeplum & CBS 119004 & КС968907 & KY610445 & KY624255 & КС977268 & $\begin{array}{l}\text { Kuhnert et } \\
\text { al. (2014), } \\
\text { Wendt et al. } \\
\text { (2018) }\end{array}$ \\
\hline H. fendleri & MUCL 54792 & KF234421 & KY610481 & KY624298 & KF300547 & $\begin{array}{l}\text { Kuhnert et } \\
\text { al. (2014), } \\
\text { Wendt et al. } \\
\text { (2018) }\end{array}$ \\
\hline H. fragiforme $e^{\mathbf{T}}$ & MUCL 51264 & KC477229 & KM186295 & KM186296 & KX271282 & $\begin{array}{l}\text { Stadler et al. } \\
\text { (2014), } \\
\text { Daranagama } \\
\text { et al. (2015) } \\
\text {,Wendt et al. } \\
\text { (2018) }\end{array}$ \\
\hline H. fuscum ${ }^{\mathbf{T}}$ & CBS 113049 & KY610401 & KY610482 & KY624299 & KX271271 & $\begin{array}{l}\text { Wendt et al. } \\
\text { (2018) }\end{array}$ \\
\hline $\begin{array}{l}\text { H. } \\
\text { griseobrunneum }^{\mathbf{T}}\end{array}$ & CBS 331.73 & KY610402 & KY610483 & KY624300 & КС977303 & $\begin{array}{l}\text { Kuhnert et } \\
\text { al. (2014), } \\
\text { Wendt et al. } \\
\text { (2018) }\end{array}$ \\
\hline $\begin{array}{l}\text { H. } \\
\text { haematostroma }\end{array}$ & MUCL 53301 & КС968911 & KY610484 & KY624301 & КС977291 & $\begin{array}{l}\text { Kuhnert et } \\
\text { al. (2014), } \\
\text { Wendt et al. } \\
\text { (2018) }\end{array}$ \\
\hline H. howeanum & MUCL 47599 & AM749928 & KY610448 & KY624258 & KC977277 & $\begin{array}{l}\text { Bitzer et al. } \\
\text { (2008), } \\
\text { Kuhnert et } \\
\text { al. (2014), } \\
\text { Wendt et al. } \\
\text { (2018) }\end{array}$ \\
\hline H. hypomiltum & MUCL 51845 & KY610403 & KY610449 & KY624302 & KX271249 & $\begin{array}{l}\text { Wendt et al. } \\
\text { (2018) }\end{array}$ \\
\hline
\end{tabular}


Table 1 Continued.

\begin{tabular}{|c|c|c|c|c|c|c|}
\hline \multirow[t]{2}{*}{ Species } & \multirow{2}{*}{$\begin{array}{l}\text { Strain } \\
\text { number }\end{array}$} & \multicolumn{4}{|c|}{ GenBank accession numbers } & \multirow[t]{2}{*}{ References } \\
\hline & & ITS & LSU & RPB2 & BTUB & \\
\hline H. investiens & CBS 118183 & KC968925 & KY610450 & KY624259 & KC977270 & $\begin{array}{l}\text { Kuhnert et } \\
\text { al. (2014), } \\
\text { Wendt et al. } \\
\text { (2018) }\end{array}$ \\
\hline $\begin{array}{l}H . \\
\text { lateripigmentum }^{\mathbf{T}}\end{array}$ & MUCL 53304 & КС968933 & KY610486 & KY624304 & КС977290 & $\begin{array}{l}\text { Kuhnert et } \\
\text { al. (2014), } \\
\text { Wendt et al. } \\
\text { (2018) }\end{array}$ \\
\hline H. lenormandii & CBS 119003 & КС968943 & KY610452 & KY624261 & КС977273 & $\begin{array}{l}\text { Kuhnert et } \\
\text { al. (2014), } \\
\text { Wendt et al. } \\
\text { (2018) }\end{array}$ \\
\hline H. monticulosum ${ }^{\mathbf{T}}$ & MUCL 54604 & KY610404 & KY610487 & KY624305 & KX271273 & $\begin{array}{l}\text { Wendt et al. } \\
\text { (2018) }\end{array}$ \\
\hline Н. тизсеит & MUCL 53765 & КС968926 & KY610488 & KY624306 & КС977280 & $\begin{array}{l}\text { Kuhnert et } \\
\text { al. (2014), } \\
\text { Wendt et al. } \\
\text { (2018) }\end{array}$ \\
\hline H. papillatum ${ }^{\mathbf{T}}$ & ATCC 58729 & КС968919 & KY610454 & KY624223 & КС977258 & $\begin{array}{l}\text { Kuhnert et } \\
\text { al. (2014), } \\
\text { Wendt et al. } \\
\text { (2018) }\end{array}$ \\
\hline H. perforatum & CBS 115281 & KY610391 & KY610455 & KY624224 & KX271250 & $\begin{array}{l}\text { Wendt et al. } \\
\text { (2018) }\end{array}$ \\
\hline H. petriniae ${ }^{\mathrm{T}}$ & CBS 114746 & KY610405 & KY610491 & KY624279 & KX271274 & $\begin{array}{l}\text { Kuhnert et } \\
\text { al. (2014), } \\
\text { Wendt et al. } \\
\text { (2018) }\end{array}$ \\
\hline H. porphyreum & CBS 119022 & КС968921 & KY610456 & KY624225 & KC977264 & $\begin{array}{l}\text { Kuhnert et } \\
\text { al. (2014), } \\
\text { Wendt et al. } \\
\text { (2018) }\end{array}$ \\
\hline H. pulicicidum ${ }^{\mathbf{T}}$ & CBS 122622 & JX183075 & KY610492 & KY624280 & JX183072 & $\begin{array}{l}\text { Bills et al. } \\
\text { (2012), } \\
\text { Wendt et al. } \\
\text { (2018) }\end{array}$ \\
\hline H. rubiginosum ${ }^{\mathbf{T}}$ & MUCL 52887 & KC477232 & KY610469 & KY624266 & KY624311 & $\begin{array}{l}\text { Stadler et al. } \\
\text { (2013), } \\
\text { Wendt et al. } \\
\text { (2018) }\end{array}$ \\
\hline H. samuelsii ${ }^{\mathbf{T}}$ & MUCL 51843 & КС968916 & KY610466 & KY624269 & KC977286 & $\begin{array}{l}\text { Kuhnert et } \\
\text { al. (2014), } \\
\text { Wendt et al. } \\
\text { (2018) }\end{array}$ \\
\hline $\begin{array}{l}\text { H. } \\
\text { submonticulosum }^{\mathbf{T}}\end{array}$ & CBS 115280 & КС968923 & KY610457 & KY624226 & КC977267 & $\begin{array}{l}\text { Kuhnert et } \\
\text { al. (2014), } \\
\text { Wendt et al. } \\
\text { (2018) }\end{array}$ \\
\hline
\end{tabular}


Table 1 Continued.

\begin{tabular}{|c|c|c|c|c|c|c|}
\hline \multirow[t]{2}{*}{ Species } & \multirow{2}{*}{$\begin{array}{l}\text { Strain } \\
\text { number }\end{array}$} & \multicolumn{4}{|c|}{ GenBank accession numbers } & \multirow[t]{2}{*}{ References } \\
\hline & & ITS & LSU & RPB2 & BTUB & \\
\hline H ticinense & CBS 115271 & JQ009317 & KY610471 & KY624272 & AY951757 & $\begin{array}{l}\text { Hsieh et al. } \\
\text { (2005), } \\
\text { Wendt et al. } \\
\text { (2018) }\end{array}$ \\
\hline H. trugodes ${ }^{\mathbf{T}}$ & MUCL 54794 & KF234422 & KY610493 & KY624282 & KF300548 & $\begin{array}{l}\text { Kuhnert et } \\
\text { al. (2014), } \\
\text { Wendt et al. } \\
\text { (2018) }\end{array}$ \\
\hline $\begin{array}{l}\text { Jackrogersella } \\
\text { multiformis }^{\mathrm{T}}\end{array}$ & CBS 119016 & KC477234 & KY610473 & KY624290 & KX271262 & $\begin{array}{l}\text { Kuhnert et } \\
\text { al. (2014), } \\
\text { Kuhnert et } \\
\text { al. (2016), } \\
\text { Wendt et al. } \\
\text { (2018) }\end{array}$ \\
\hline $\begin{array}{l}\text { Pyrenopolyporus } \\
\text { nicaraguensis }\end{array}$ & CBS 117739 & AM749922 & KY610489 & KY624307 & КС977272 & $\begin{array}{l}\text { Bitzer et al. } \\
\text { (2008), } \\
\text { Wendt et al. } \\
\text { (2018) }\end{array}$ \\
\hline $\begin{array}{l}\text { Rhopalostroma } \\
\text { angolense }\end{array}$ & CBS 126414 & KY610420 & KY610459 & KY624228 & KX271277 & $\begin{array}{l}\text { Wendt et al. } \\
\text { (2018) }\end{array}$ \\
\hline $\begin{array}{l}\text { Rostrohypoxylon } \\
\text { terebratum }^{\mathbf{T}}\end{array}$ & CBS 119137 & DQ631943 & DQ840069 & DQ631954 & DQ840097 & $\begin{array}{l}\text { Tang et al. } \\
(2007) \text {, } \\
\text { Fournier et } \\
\text { al. (2010) }\end{array}$ \\
\hline $\begin{array}{l}\text { Ruwenzoria } \\
\text { pseudoannulata }^{\mathbf{T}}\end{array}$ & MUCL 51394 & KY610406 & KY610494 & KY624286 & KX271278 & $\begin{array}{l}\text { Wendt et al. } \\
\text { (2018) }\end{array}$ \\
\hline $\begin{array}{l}\text { Thamnomyces } \\
\text { dendroidea }\end{array}$ & CBS 123578 & FN428831 & KY610467 & KY624232 & KY624313 & $\begin{array}{l}\text { Stadler et al. } \\
\text { (2010), } \\
\text { Wendt et al. } \\
\text { (2018) }\end{array}$ \\
\hline $\begin{array}{l}\text { Xylaria hypoxylon } \\
\mathbf{T}\end{array}$ & CBS 122620 & KY610407 & KY610495 & KY624231 & KX271279 & $\begin{array}{l}\text { Wendt et al. } \\
\text { (2018) }\end{array}$ \\
\hline X. polymorpha & MUCL 49884 & KY610408 & KY610464 & KY624288 & KX271280 & $\begin{array}{l}\text { Wendt et al. } \\
\text { (2018) }\end{array}$ \\
\hline
\end{tabular}

*ATCC: American Type Culture Collection, Virginia, USA: CBS: Centraalbureau voor Schimmelcultures, Utrecht, The Netherlands, KC: Kew Culture Collection, United Kingdom, MFLUCC: Mae Fah Luang University Culture Collection, Chiang Rai, Thailand, MUCL: Université Catholique de Louvain, Belgium, STMA: Personal Herbarium and Culture Collection of M. Stadler.

\section{Phylogenetic analysis}

Maximum likelihood (ML) trees were generated using the RAxML-HPC2 on XSEDE (8.2.8) (Stamatakis et al. 2008, Stamatakis 2014) in the CIPRES Science Gateway platform (Miller et al. 2010) using GTR +I+G model of evolution.

A Bayesian analysis was conducted with MrBayes v. 3.1.2 (Huelsenbeck \& Ronqvist 2001) to evaluate Posterior probabilities (PP) (Rannala \& Yang 1996, Zhaxybayeva \& Gogarten 2002) by Markov Chain Monte Carlo sampling (BMCMC). Two parallel runs were conducted, using the default settings, but with the following adjustments: Four simultaneous Markov chains were run for 2,000,000 generations and trees were sampled every 100th generation and 20,000 trees were 
obtained. The first 4,000 trees, representing the burnin phase of the analyses and discarded. The remaining 16,000 trees were used for calculating PP in the majority rule consensus tree.

Phylograms were visualized with FigTree v1.4.0 program (Rambaut 2011) and reorganized in Microsoft power point.

\section{Results}

\section{Phylogenetic analysis of $D$. eschscholtzii}

The combined LSU, ITS, RPB2 and BTUB matrix comprised 76 sequences including selected genera in Hypoxylaceae, D. eschscholtzii (MFLUCC18-0177, MFLUCC19-0154, MFLUCC19-0153) strains and Xylaria hypoxylon (CBS 122620) and X. polymorpha (MUCL 49884) (Xylariaceae) as out group taxa (Fig. 1). Four different alignments of each individual gene and a combined alignment of four genes were analyzed in this study. A best scoring RAxML tree is shown in Fig. 1 with a final ML optimization likelihood value of -35661.5741 . The matrix had 1340 distinct alignment patterns, with $28.10 \%$ of undetermined characters or gaps. Estimated base frequencies were as follows; $A=0.246955, C=0.244469, G=0.266113, T=0.242464$; substitution rates $\mathrm{AC}=1.597317, \mathrm{AG}=4.770781, \mathrm{AT}=1.560157, \mathrm{CG}=1.203822, \mathrm{CT}=7.713893, \mathrm{GT}=1.000000$; proportion of invariable sites $\mathrm{I}=0.466559$; gamma distribution shape parameter $\alpha=0.816750$. All trees (ML and BYPP) were similar in topology and did not differ significantly (data not shown) at the generic relationships which is in agreement with previous study based on multi-gene phylogeny of Stadler et al. (2014) and Wendt et al. (2018). Bootstrap support (BS) values of ML (equal or greater than $60 \%$ based on 1000 replicates) are shown on the upper branches with blue. Branches with Bayesian posterior probabilities (PP) greater than 0.95 from MCMC analyses are given in blue. Our isolates (MFLUCC18-0177, MFLUCC19-0154, MFLUCC19-0153) were clustered with in $D$. eschscholtzii with a significant bootstrap support $(\mathrm{ML}=100 \%, \mathrm{PP}=1.00)$.

\section{Taxonomy}

Daldinia eschscholtzii (Ehrenb.) Rehm, Annls mycol. 2(2): 175 (1904)

Fig. 2 Index Fungorum Number: IF544992; Facesoffungi Number: FoF02990

Endophytic on fresh leaves of Musa sp. Colonies on PDA at $25^{\circ} \mathrm{C}$ temperature, light, reaching $7 \mathrm{~cm}$ in two weeks, initially white with a diffuse margin. Colonies become gray, with olive green and later become dull green spots. When mature purple pigmentation occurs from the center of the colony. Sexual morph: Stromatic structures arising from the surface after 8 weeks, convexly curved, swelled, sterile. Asexual morph: Sporulation after two to three weeks, under $25^{\circ} \mathrm{C}$, entire surface after three weeks, becomes gray after four weeks, reverse black at the center and whitish gray at the periphery after two weeks. Mycelium 1-3.5 $\mu \mathrm{m}(\overline{\mathrm{x}}=2.75 \mu \mathrm{m})$ wide, superficial, composed of septate, branched, rough, inflated, often have melanized hyphae with brownish exudates in old cultures. Conidiophores 0.8-2 $\mu \mathrm{m}$ long $\times 0.7-1.5 \mu \mathrm{m}$ diam. $(\overline{\mathrm{x}}=1.5 \times 1.3 \mu \mathrm{m}, \mathrm{n}=10)$ hyaline, mononematous synonymous, short, small, conidiogenous structure dichotomous or trichotomous with nodulisporium-like branching pattern, 1-3 conidiogenous cells arise from each terminus. Conidiogenous cells $2.8-4 \times 1.7-5 \mu \mathrm{m}(\overline{\mathrm{x}}=3.4 \times 2.1 \mu \mathrm{m}, \mathrm{n}=10)$, hyaline, holoblastic, terminal or intercalary, cylindrical, having rounded apices, texture, collaret or opening width. Conidia 3-5.5 $\mu \mathrm{m} \times 2-3.5 \mu \mathrm{m}(\overline{\mathrm{x}}=4.6 \times 2.5 \mu \mathrm{m}, \mathrm{n}=40)$, obovoid to ellipsoid, aseptate, hyaline, smooth often flat at the base.

Materials examined - THAILAND, Chiang Rai, in symptomless fresh leaves of Musa sp., 15 October 2017, BC. Samarakoon, BN021 (5) (MFLU 19-0409), living culture MFLUCC 18-0177. Phayao, in symptomless fresh leaves of Musa sp., 23 January 2018, BC. Samarakoon, BNE003 (MFLU 19-0408), living culture MFLUCC 19-0154. Phu Chi Fah, in symptomless fresh leaves of Musa sp. 17 March 2018, BC. Samarakoon, BNE002 (MFLU 19-0407), living culture MFLUCC 19-0153. 


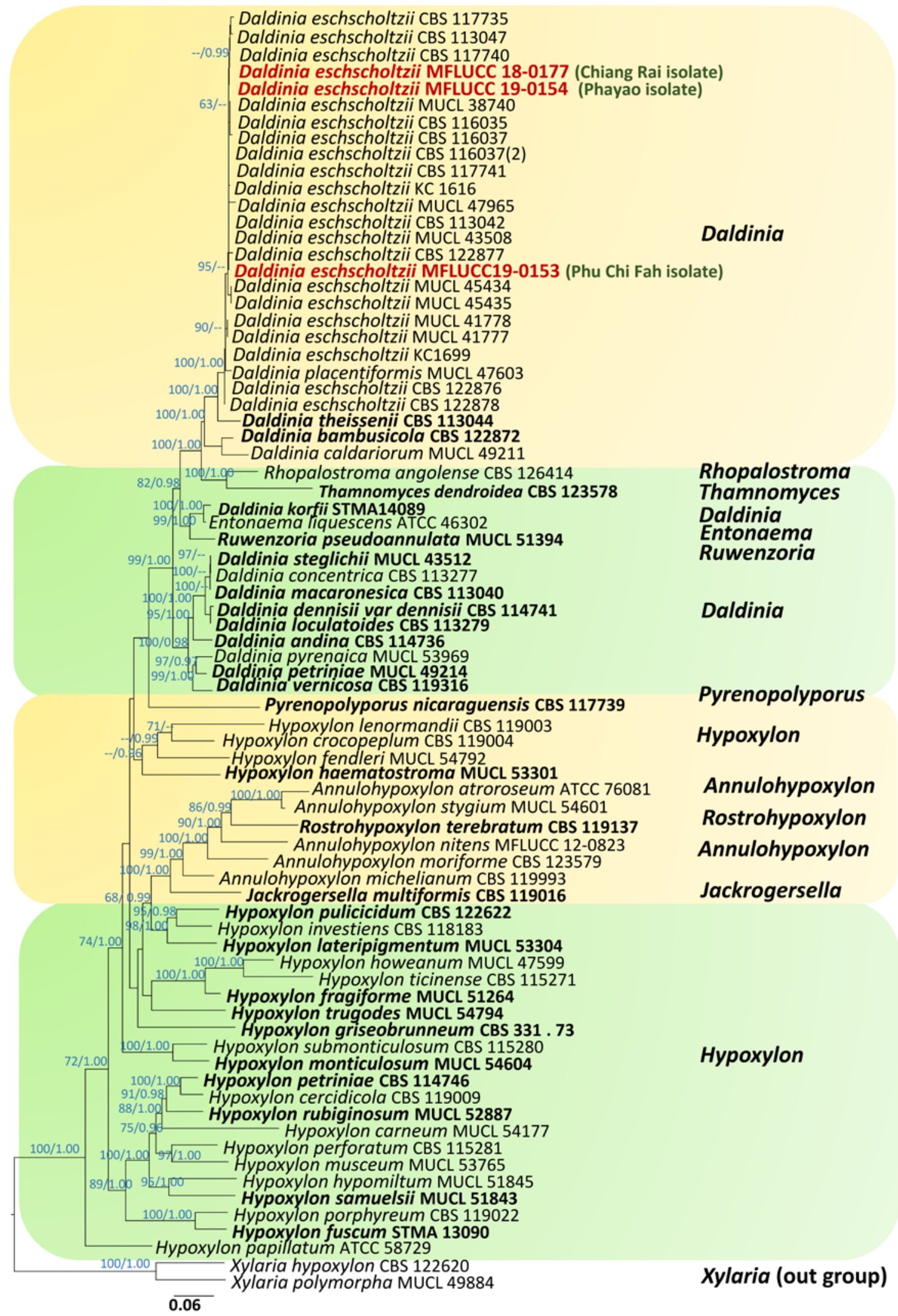

Fig. 1 - Maximum likelihood tree revealed by RAxML from an analysis of combined LSU-ITSRPB2 and BTUB rDNA matrix of Daldinia, showing the phylogenetic position of $D$. eschscholtzii. ML bootstrap supports $(\geq 60 \%)$ and Bayesian posterior probabilities $(\geq 0.95 \mathrm{PP})$ are given above in the branches respectively. The tree was rooted with Xylaria polymorpha and $X$. hypoxylon (Xylariaceae). Strain generated in this study is indicated in red-boldface, and type strains are in black-boldface. The scale bar represents the expected number of nucleotide substitutions per site. 

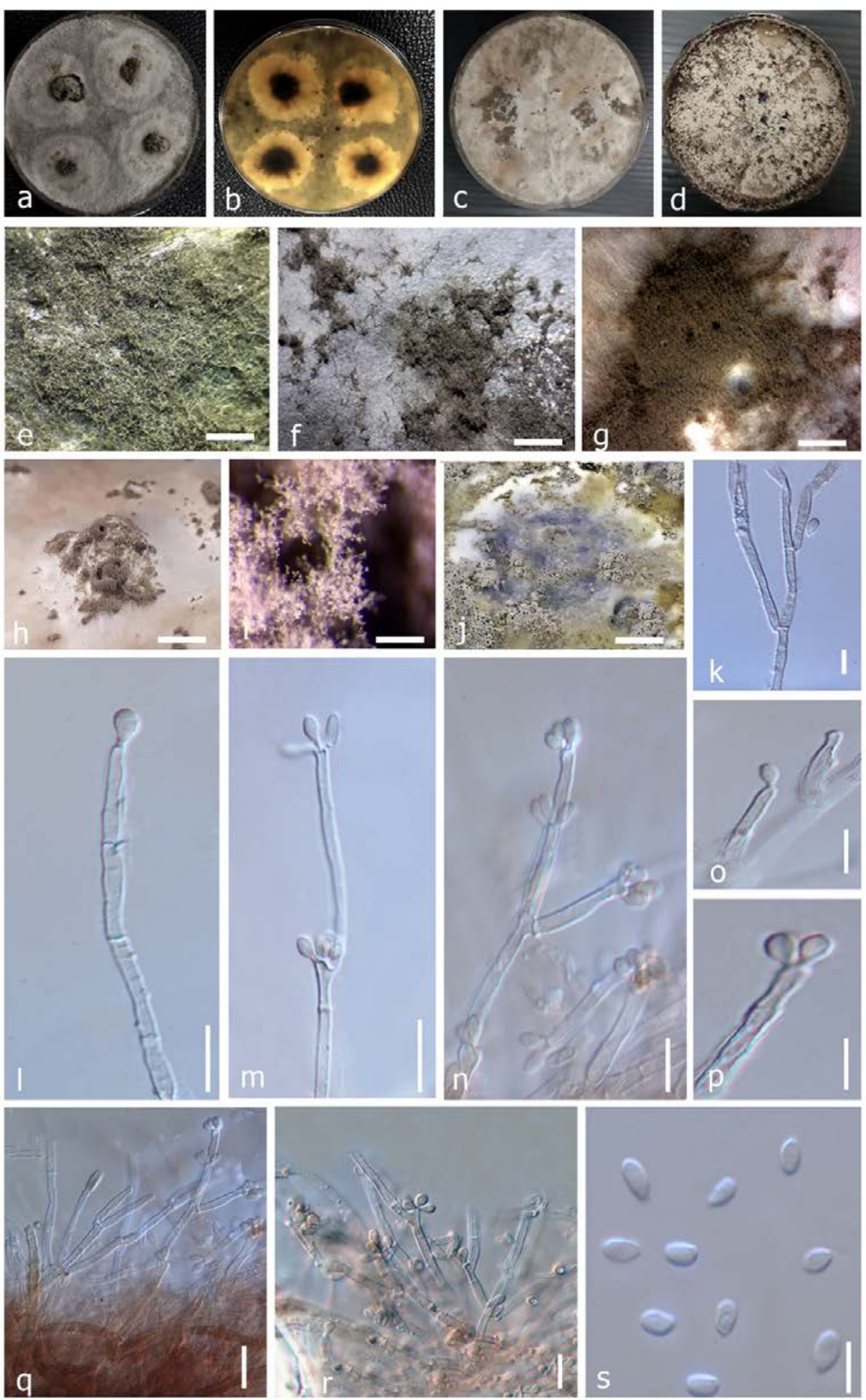

Fig. 2 - Daldinia eschscholtzii. a, b immature colony on PDA after 3 weeks. c Immature colony on PDA after 2 weeks. d Colonies on PDA after 8 weeks bearing sterile stromatic structures showing sporulation in mouse gray. e greenish patches on PDA. f-i Sporulation of the colony showing conidiomata on PDA. j Pigmentation on PDA and sterile stromatic structures. k Mycelium with nodulisporium-like branching pattern. $\mathrm{l}$, m Conidial attachments and conidiogenous cells showing sporothrix-like branching pattern in the mycelium. n-r Conidial attachments and conidiogenous cells showing nodulisporium-like branching pattern in the mycelium s Conidia. a, b, e, f, j, k, l-r from MFLUCC 18-0177; c, g, h, i from MFLUCC 19-0154; d from MFLUCC 19-0153. Scale bars: $\mathrm{e}-\mathrm{j}=200 \mu \mathrm{m}, \mathrm{k}-\mathrm{r}=10 \mu \mathrm{m}, \mathrm{s}=2 \mu \mathrm{m}$ 


\section{Discussion}

Studies about musaceous endophytes in Thailand were conducted by Photita et al. (2001, 2004) who isolated 61 fungal taxa from Doi Suthep Pui National Park, but did not conduct any molecular justification. To address this research gap, we are reinvestigating the fungi on Musa sp. in different geographic regions around the country, isolate them into a culture, and describe their morphology and phylogenetic relationships. During our study so far, we have isolated many taxa that have previously been recorded in Photita et al. (2001, 2004) with confirmed molecular data (not shown).

Few studies found that nonpathogenic endophytic strains of Fusarium oxysporum Schltdl. that have been isolated from healthy banana rhizomes can induce systemic resistance of the plant against Radopholus similis (pathogenic root nematode) in Musa sp. (Vu et al. 2006). In addition, endophytic $F$. oxysporum strains have controlled the nematode reproduction and damage (Sikora et al. 2008) and acted as potential growth promoters in Musa sp. (Ting et al. 2008). Therefore, the role of endophytic fungi in Musa sp. should be further investigated.

Daldinia graminis Dargan \& K.S. Thind and D. sacchari Dargan \& K.S. Thind was recorded from sugarcane plant in India. In addition D. bambusicola Y.M. Ju, J.D. Rogers \& F. San Martín was found from bamboo in Thailand (Stadler et al. 2014). As a conclusion, four Daldinia species have so far been found on monocots including D. eschscholtzii.

Production of stromata is a characteristic feature of all the daldinoid clades in Hypoxylaceae. Generally, in natural habitats, the stromatic structures support the fungi to survive in harsh environmental conditions (Stadler et al. 2014). The stromata of D. eschscholtzii produce bioactive compounds (secondary metabolites) such as binaphthalene derivatives (BNT), cytochalasins and concentricols (Stadler et al. 2014, Zhang et al. 2008, 2011). In addition Helaly et al. (2018) have documented, a mantis-associated culture of $D$. eschscholtzii produces dalesconols and spirodalesol, which have strong immunomodulatory effects. Interestingly our asexual cultures of $D$. eschscholtzii isolates had a limited lifespan compared to the other endophytic isolates. This can be due to the self-poisoning of the cultures as a result of releasing antibiotic compounds (Stadler et al. 2014). Therefore, future studies will be rather warranted to extract the bioactive compounds from our $D$. eschscholtzii isolates and investigate the functions of the compounds on the host or commercial industry.

\section{Acknowledgements}

The authors like to acknowledge the National Research Council of Thailand (Grant No. 256108A3070006) and Mae Fah Luang University (Grant No. DR256201012003) for the financial support. Dhanushka Wanasinghe would like to thank CAS President's International Fellowship Initiative (PIFI) for funding his postdoctoral research (number 2019PC0008), the National Science Foundation of China and the Chinese Academy of Sciences for financial support under the following grants: 41761144055, 41771063 and Y4ZK111B01. We would like to thank Kevin D. Hyde, Q. Tian, Chuangen Lin, W.A.E. Yasanthika, C.S. Bhunjun and G. Samarakoon for the valuable suggestions they have made.

\section{References}

Bills GF, Gonzalez-MenendezV MJ, Platas G, Fournier J et al. 2012 - Hypoxylon pulicicidum sp. nov. (Ascomycota, Xylariales), a pantropical insecticide-producing endophyte. PLoS One 7:e46687

Bitzer J, Læssøe T, Fournier J, Kummer V et al. 2008 - Affinities of Phylacia and the daldinoid Xylariaceae, inferred from chemotypes of cultures and ribosomal DNA sequences. Mycological Research 112:251-270.

Brown KB, Hyde KD, Guest DI. 1998 - Preliminary studies on endophytic fungal communities of Musa acuminata species complex in Hong Kong and Australia. Fungal Diversity 1, 27-51. 
Cao LX, Tian XL, Zhou SN. 2003 - Isolation of endophytic fungi and Actinomycetes from banana (Musa paradisiaca) plants. Acta Scientiarum Naturalium Universitatis Sunyatseni 2, 20-23.

Cao LX, You JL, Zhou SN. 2002 - Endophytic fungi from Musa acuminata leaves and roots in South China. World Journal of Microbiology and Biotechnology 18, 169-171. https://doi.org/10.1023/A:1014491528811

Cesati V, De Notaris G. 1863 - Schema di classificazione degle sferiacei italici aschigeri piu’ o meno appartenenti al genere Sphaeria nell'antico significato attribuitoglide Persono. Commentario della Società Crittogamologica Italiana 1, 177-420.

Daranagama DA, Camporesi E, Tian Q, Liu X et al. 2015 - Anthostomella is polyphyletic comprising several genera in Xylariaceae. Fungal Diversity 73, 203-238.

Fournier J, Stadler M, Hyde KD, Duong LM. 2010 - The new genus Rostrohypoxylon and two new Annulohypoxylon species from Northern Thailand. Fungal Diversity 40, 23-36.

Guidot A, Johannesson H, Dahlberg A, Stenlid J. 2003 - Parental tracking in the postfire wood decay ascomycete Daldinia loculata using highly variable nuclear gene loci. Molecular Ecology 12, 1717-1730. https://doi.org/10.1046/j.1365-294X.2003.01858.x

Hall TA. 1999 - BioEdit: a user-friendly biological sequence alignment editor and analysis program for Windows 95/98/NT. Nucleic Acids Symposium Series 41, 95-98. Available from: http://www.mbio.-ncsu.edu/bioedit/bioedit.html

Helaly SE, Thongbai B, Stadler M. 2018 - Diversity of biologically active secondary metabolites from endophytic and saprotrophic fungi of the ascomycete order Xylariales. Natural product reports 35, 992-1014.

Hsieh HM, Ju YM, Rogers JD. 2005 - Molecular phylogeny of Hypoxylon and closely related genera. Mycologia 97, 844-865.

Huelsenbeck JP, Ronquist F. 2001 - MRBAYES: Bayesian inference of phylogenetic trees. Bioinformatics 17, 754-755. https://doi.org/10.1093/bioinformatics/17.8.754

Hyde KD, Norphanphoun C, Chen J, Dissanayake AJ et al. 2018 - Thailand's amazing diversity: up to $96 \%$ of fungi in northern Thailand may be novel. Fungal Diversity 93, 215-239. https://doi.org/10.1007/s13225-018-0415-7

Jayawardena RS, Hyde KD, Jeewon R, Ghobad-Nejhad M et al. 2019 - One stop shop II: taxonomic update with molecular phylogeny for important phytopathogenic genera: 26-50. Fungal Diversity 94, 41-129. https://doi.org/10.1007/s13225-019-00418-5

Johannesson H, Laessøe T, Stenlid J. 2000 - Molecular and morphological investigation of the genus Daldinia in Northern Europe. Mycological Research 104, 275-280.

Johannesson H, Vasiliauskas R, Dahlberg A, Penttilä R, Stenlid J. 2001 - Genetic differentiation in Eurasian populations of the postfire ascomycete Daldinia loculata. Molecular Ecology 10, 1665-1677. https://doi.org/10.1046/j.1365-294X.2001.01317.x

Karnchanatat A, Petsom A, Sangvanich P, Piaphukiew J et al. 2007 - Purification and biochemical characterization of an extracellular beta-glucosidase from the wood-decaying fungus Daldinia eschscholzii (Ehrenb.:Fr.) Rehm. FEMS Microbiology Letters 270, 162-70. https://doi.org/10.1111/j.1574-6968.2007.00662.x

Katoh K, Rozewicki J, Yamada KD. 2017 - MAFFT online service: multiple sequence alignment, interactive sequence choice and visualization. Briefings in Bioinformatics. bbx108. https://doi.org/10.1093/bib/bbx108

Kongyen W, Rukachaisirikul V, Phongpaichit S, Sakayaroj J. 2015 - A new hydronaphthalenone from the mangrove-derived Daldinia eschscholtzii PSU-STD57. Natural Product Research 29, 1995-1999. https://doi.org/10.1080/14786419.2015.1022542

Kuhnert E, Fournier J, Peršoh D, Luangsa-ard JJD, Stadler M. 2014 - New Hypoxylon species from Martinique and new evidence on the molecular phylogeny of Hypoxylon based on ITS rDNA and $\beta$-tubulin data. Fungal Diversity 64,181-203.

Liu YJ, Whelen S, Hall BD. 1999 - Phylogenetic relationships among ascomycetes: evidence from an RNA polymerse II subunit. Molecular Biology and Evolution 16, 1799-1808. https://doi.org/10.1093/oxfordjournals.molbev.a026092 
Miller MA, Pfeiffer W, Schwartz T. 2010 - Creating the CIPRES science gateway for inference of large phylogenetic trees. Proceedings of the Gateway Computing Environments Workshop (GCE), November 14, 2010, New Orleans, Louisiana 1-8. https://doi.org/10.1109/GCE.2010.5676129

Ng KP, Chan CL, Yew SM, Yeo SK et al. 2016 - Identification and characterization of Daldinia eschscholtzii isolated from skin scrapings, nails, and blood. PeerJ 4, e2637. https://doi.org/10.7717/peerj.2637

Nugent LK. 2004 - Latent invasion by Xylariaceous fungi. PhD Thesis. Liverpool John Moores University, Liverpool, UK.

O’Donnell K, Cigelnik E. 1997 - Two divergent intragenomic rDNA ITS2 types within a monophyletic lineage of the fungus Fusarium are nonorthologous. Molecular Phylogenetics and Evolution 7, 103-116. https://doi.org/10.1006/mpev.1996.0376

Pereira JO, Vieira MC, Azevedo JL. 1999 - Endophytic fungi from Musa acuminata and their reintroduction into axenic plants. World Journal of Microbiology and Biotechnology 15, 3740. https://doi.org/10.1023/A:1008859823806

Photita W, Lumyong S, Lumyong P. 2001 - Endophytic fungi of wild banana (Musa acuminata) at Doi Suthep Pui National Park, Thailand. Mycological Research 105, 1508-1513. https://doi.org/10.1017/S0953756201004968

Photita W, Lumyong S, Lumyong P, McKenzie EHC, Hyde KD. 2004 - Are some endophytes of Musa acuminata latent pathogens? Fungal Diversity 16, 131-140.

Pocasangre L, Sikora RA, Vilich V, Schuster RP. 1999 - Survey of banana endophytic fungi from Central America and screening for biological control of Radopholus similis. In II ISHS Conference on Fruit Production in the Tropics and Subtropics 531, 283-290. https://doi.org/10.17660/ActaHortic.2000.531.47

Rambaut A. 2011 - FigTree. Tree figure drawing tool version 1.3.1, Institute of Evolutionary Biology, University of Edinburgh. Available from: http://tree.bio.ed.ac.uk/software/figtree/ (accessed 20 June 2019)

Rannala B, Yang Z. 1996 - Probability distribution of molecular evolutionary trees: a new method of phylogenetic inference. Journal of Molecular Evolution 43, 304-311. https://doi.org/10.1007/BF02338839

Schulz B, Boyle C. 2006 - What are endophytes? In Microbial root endophytes pp. 1-13. Springer, Berlin, Heidelberg. https://doi.org/10.1007/3-540-33526-9_1

Sikora RA, Pocasangre L, zum Felde A, Niere B et al. 2008 - Mutualistic endophytic fungi and inplanta suppressiveness to plant parasitic nematodes. Biological Control 46, 15-23. https://doi.org/10.1016/j.biocontrol.2008.02.011

Sir EB, Lambert C, Wendt L, Hladki AI et al. 2016 - A new species of Daldinia (Xylariaceae) from the Argentine subtropical montane forest. Mycosphere 7, 1378-1388. https://doi.org/10.5943/mycosphere/7/9/11

Stadler M, Laessoe T, Fournier J, Decock C et al. 2014 - A polyphasic taxonomy of Daldinia (Xylariaceae). Studies in Mycology 77, 1-143. https://doi.org/10.3114/sim0016

Stamatakis A. 2014 - RAxML version 8: a tool for phylogenetic analysis and post-analysis of large phylogenies. Bioinformatics 30, 1312-1313. https://doi.org/10.1093/bioinformatics/btu033

Stamatakis A, Hoover P, Rougemont J. 2008 - A rapid bootstrap algorithm for the RAxML web servers. Systematic Biology 57, 758-771. https://doi.org/10.1080/10635150802429642

Tang AM, Jeewon R, Hyde KD. 2007 - Phylogenetic relationships of Nemania plumbea sp. nov. and related taxa based on ribosomal ITS and RPB2 sequences. Mycological Research 111, 392-402.

Tibpromma S, Hyde KD, Bhat JD, Mortimer PE et al. 2018 - Identification of endophytic fungi from leaves of Pandanaceae based on their morphotypes and DNA sequence data from southern Thailand. MycoKeys 33, 25-67. https://doi.org/10.3897/mycokeys.33.23670 
Ting AS, Meon S, Kadir J, Radu S, Singh G. 2008 - Endophytic microorganisms as potential growth promoters of banana. BioControl 53, 541-553. https://doi.org/10.1007/s10526-0079093-1

Triebel D, Peršoh D, Wollweber H, Stadler M. 2005 - Phylogenetic relationships among Daldinia, Entonaema and Hypoxylon as inferred from ITS nrDNA sequences. Nova Hedwigia 80, 2543.

Vilgalys R, Hester M. 1990 - Rapid genetic identification and mapping of enzymatically amplified ribosomal DNA from several Cryptococcus species. Journal of Bacteriology 172, 4238-4246. https://doi.org/10.1128/jb.172.8.4238-4246.1990

Vu T, Sikora R, Hauschild R. 2006 - Fusarium oxysporum endophytes induced systemic resistance against Radopholus similis on banana. Nematology 8, 847-852.

https://doi.org/10.1163/156854106779799259

Wanasinghe DN, Jeewon R, Jones EG, Boonmee S et al. 2018 - Novel palmicolous taxa within Pleosporales: multigene phylogeny and taxonomic circumscription. Mycological Progress 17, 571-590. https://doi.org/10.1007/s11557-018-1379-4

Wanasinghe DN, Jones EBG, Camporesi E, Boonmee S et al. 2014 - An exciting novel member of Lentitheciaceae in Italy from Clematis vitalba. Cryptogamie Mycologie 35, 323-337. https://doi.org/10.7872/crym.v35.iss4.2014.323

Wang YU, Guo LD, Hyde KD. 2005 - Taxonomic placement of sterile morphotypes of endophytic fungi from Pinus tabulaeformis (Pinaceae) in northeast China based on rDNA sequences. Fungal Diversity 20, 235-260.

Wendt L, Sir EB, Kuhnert E, Heitkämper S et al. 2018 - Resurrection and emendation of the Hypoxylaceae, recognised from a multigene phylogeny of the Xylariales. Mycological Progress 17, 115-154. https://doi.org/10.1007/s11557-017-1311-3

White TJ, Bruns T, Lee SJWT, Taylor JL. 1990 - Amplification and direct sequencing of fungal ribosomal RNA genes for phylogenetics. PCR protocols: a guide to methods and applications 18, 315-322.

Wijayawardene NN, Hyde KD, Rajeshkumar KC, Hawksworth DL et al. 2017 - Notes for genera: Ascomycota. Fungal Diversity 86, 1-594. https://doi.org/10.1007/s13225-017-0386-0

Wongkanoun S, Wendt L, Stadler M, Luangsa-ard J, Srikitikulchai P. 2019 - A novel species and a new combination of Daldinia from Ban Hua Thung community forest in the northern part of Thailand. Mycological Progress 18, 553-564. https://doi.org/10.1007/s11557-019-01469-3

Zakaria L, Aziz W. 2018 - Molecular identification of endophytic fungi from banana leaves (Musa spp.). Tropical Life Sciences Research 29, 201-211. https://doi.org/10.21315/tlsr2018.29.2.14

Zhang YL, Ge HM, Zhao W, Dong H et al. 2008 - Unprecedented immunosuppressive polyketides from Daldinia eschscholzii, a mantis-associated fungus. Angewandte Chemie International Edition in English 47, 5823-5826. https://doi.org/10.1002/anie.200801284

Zhang YL, Zhang J, Jiang N, Lu YH et al. 2011 - Immunosuppressive polyketides from mantisassociated Daldinia eschscholzii. Journal of the American Chemical Society 133, 5931-5940. https://doi.org/ 10.1021/ja110932p

Zhaxybayeva O, Gogarten JP. 2002 - Bootstrap, Bayesian probability and maximum likelihood mapping: exploring new tools for comparative genome analyses. BMC genomics 3, 4 . https://doi.org/10.1186/1471-2164-3-4 